\title{
Tools and a web server for data analysis and presentation in microbial ecology
}

\author{
S. Feranchuk ${ }^{6}$, N. Belkova ${ }^{1}$, U. Potapova ${ }^{2}$, I. Ochirov ${ }^{3}$, D. Kuzmin ${ }^{4,5}$ and S. Belikov ${ }^{2}$ \\ ${ }^{1}$ Scientific Centre for Family Health and Human Reproduction Problems, 664003 Irkutsk, Russia \\ ${ }^{2}$ Limnological Institute, Siberian Branch of Russian Academy of Sciences, 664033 Irkutsk, Russia \\ ${ }^{3}$ Regional Medical and Sports Clinic “Zdorovie”, 664003, Irkutsk, Russia \\ ${ }^{4}$ Laboratory of Forest Genomics, Genome Research and Education Center, Siberian Federal University, 660036 \\ Krasnoyarsk, Russia \\ ${ }^{5}$ Department of High Performance Computing, Institute of Space and Information Technologies, Siberian Federal \\ University, 660074 Krasnoyarsk, Russia \\ ${ }^{6}$ Corresponding author; e-mail: feranchuk@gmail.com
}

Key words: Data analysis; Free software; Graphical presentation; Interactive visualization; Microbial ecology; Web server.

\begin{abstract}
The methods for data presentation are important in bioinformatics as data processing algorithms. The article describes the software package for the extensive analysis of tables with estimates of bacterial abundance levels in environmental samples. The package was designed to be executed in a distributed hardware environment, with powerful packages in Python in the backend and interactive front-end forms. Most of microbial ecology-specific functionality is implemented by the scikit-bio Python package, together with the other Python packages intended for big data analysis. Interactive visualisation tools are implemented by the D3.js software library, therefore, the software project is named D3b. The package is a suite of tools for the analysis of microbial ecology data implemented as a web-service and as a desktop application. It supports a substantial part of the graphical and analytical descriptions of microbial communities used in scientific publications. Source codes are available at github (sferanchuk/d3b_charts) and the on-line version of the system is accessible at d3b-charts.bri-shur.com.
\end{abstract}

Abbreviations: BIOM-Biology Observation Matrix; MDS-Multi-Dimensional Scaling; OTU-Operational Taxonomic Unit; PCA-Principal Components Analysis; RDP-Ribosomal Database Project.

\section{Introduction}

The contribution of microbiology to biological research has been highlighted with advances in sequencing technologies, as shown in Figure 1. Newly developed tools for data analysis in microbiology need to be able to be adapted to a growing number of possible interpretations of the data; the rich variety of tasks requires tools to address an almost continuous stream of questions.

Amplicon gene sequencing remains one of the most important methods to study microbial communities (Segata et al. 2013, Boughner and Singh 2016, Hugerth and Andersson 2017), and the conventional bioinformatics pipelines, used to process sequencing data in these studies, can be roughly divided into two stages: a resource-consuming stage of sequence clustering and taxonomic annotation, and different types of analysis and interpretation, including diversity calculations, hypothesis testing, and data visualisation. The first stage, considered in details, is subdivided into picking the amplified fragments of the reference gene into operational taxonomic units (OTU), and the assignment of the taxonomic annotation to each OTU. In the conventional systems of mi- crobial taxonomy, seven levels are used to annotate the bacteria: phylum, class, order, family, genus, and species. The annotation of OTUs might be not precise enough and identification might be limited to a genus or even to a higher level of the hierarchy (Almeida et al. 2018).

The most informative experiments include simultaneous processing of several samples. Therefore, the results from the first stage of pipeline can usually be presented in a relatively compact form of abundance tables for taxonomic units, i.e., the number of reads included in each OTU for each of the samples. This tabular data is supported by standardised formats, such as the "Biology observation matrix" (BIOM) format (McDonald et al. 2012). In addition, for the whole metagenome and metatranscriptome sequencing, software packages such as sortmerna (Kopylova et al. 2016) enable the representation of the microbiome community composition under study in the form of a table of abundance counts.

The integrated downstream pipeline with all the conventional types of data analysis and visualisation (such as diversity estimates, heatmaps, and PCA charts) could be useful to present a wide range of experiments. However, in some situations, the scientist should find a clue to the interpretation 

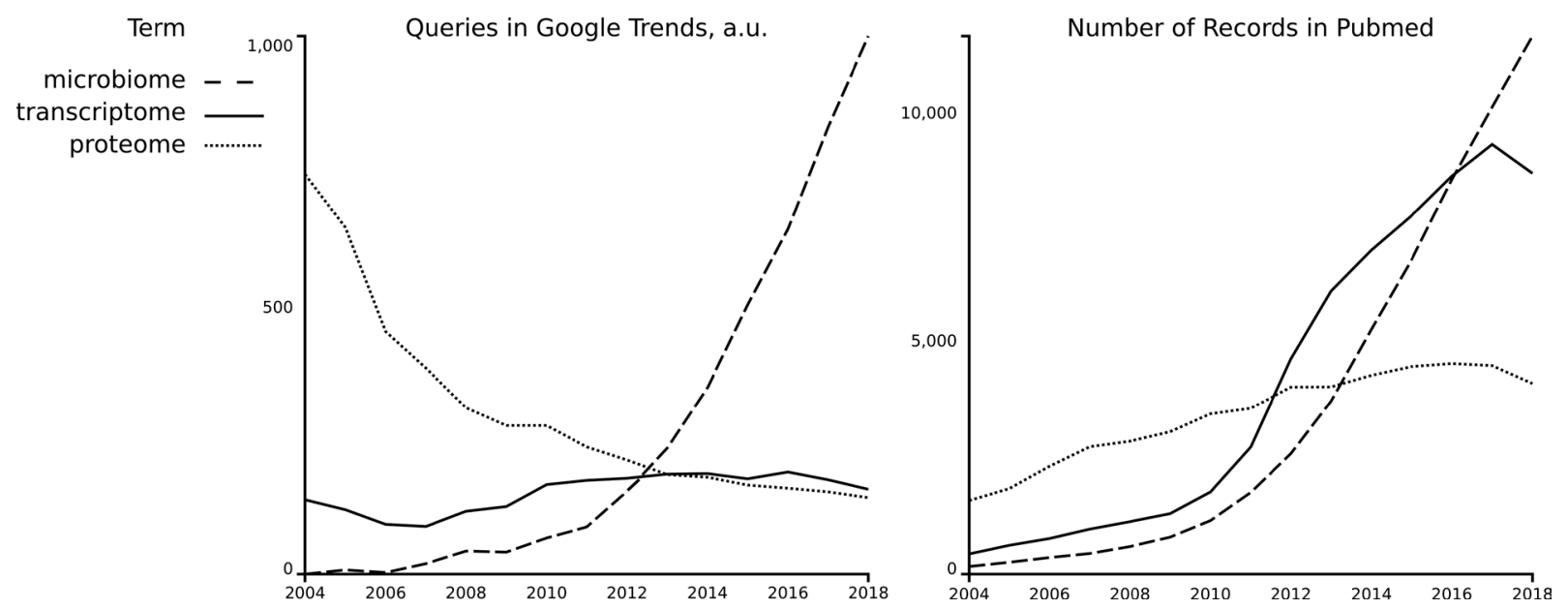

Figure 1. The growth in interest related to analysis of the microbiome, demonstrated using a chart provided by Google Trends (left) and the annual number of publications in PubMed (right).

of the experiment, and the interactive flexible tools for data presentation could help in this task.

Unification of mathematical concepts from different areas of science could be implemented within a single software infrastructure such as Python, so that the software interface for the Euclidean measure in geometry and Hamming distance in informatics could be almost the same as the BrayCurtis and Jaccard dissimilarity measures in ecology, by using the 'scipy.spatial.distance' Python library. This opens the possibility to satisfy the declared requirements of wide flexibility of data analysis tools for microbiologists. Expressive and clear graphics contribute significantly to clarifying the interpretations of the data, however, software tools, developed to manage graphics objects at a sufficiently high level of abstraction, also allow the results to be represented in the most convenient ways.

The other side to the observed development and unification of software libraries is, firstly, the care taken to ensure the mutual consistency of heterogeneous packages which are composed together to support the wide spectrum of representations at another end of a software system. A sign that the problem of inconsistencies is growing is development of packaging systems, such as Anaconda, at an increasing rate. Furthermore, the scarcity of resources, directed to software development and to the teaching of newly developed tools, leads to problems, such as competition and increased inequality in the allocation of that resource, revealing the problems typical of the most of societies, even with an increased contrast.

The list of software packages developed for microbiologists, presented below as an overview of related projects, is definitely incomplete. However, several important features could be stressed which could in some way characterise all of the listed packages. First, many of the packages are designed to be incorporated into a wide software infrastructure, such as the $\mathrm{C}++, \mathrm{R}$, or Python environment. Second, the most important and costly effort, which accomplishes the develop- ment of a package with sufficient usability, is the care taken to ensure the mutual consistency of component libraries. It is achieved either when software tools are provided as web-services (RDP (Cole et al. 2014)), or are incorporated into a single binary, with source codes which are mostly independent from auxiliary libraries (Mothur (Schloss et al. 2009)). In the QIIME project (Caporaso et al. 2010), the balance is achieved when several robust core pipelines are implemented, supported by specialised Python libraries and uclust software, where elaborated algorithms are incorporated into the pipelines. This software project allows easy incorporation of additional expansions, such as sortmerna/sumaclust. Consistency of the project is supported by distribution within the anaconda packaging system. Just as the QIIME project effectively uses the advantages of the development within the Python environment, the vegan project (Oksanen et al. 2007) uses advantages of the development within an R environment, with ability to use general-purpose tools from data science. And, aside from the microbiology software, two long-lasting projects for visualisation in structural bioinformatics, UCSF Chimera (Pettersen et al. 2004), and Pymol (The PyMOL molecular graphics system, 2017), use advantages of the development within a Python environment.

Since the volume of expected traffic and computational costs in the downstream analysis are relatively low, webbased tools are well-suited for implementation of the user interface in the interactive data presentation system. JavaScript and node.js packaging systems could be considered as an infrastructure to implement efficient online visualisation tools. The Biojs project (Yachdav et al. 2015) is an example of the general-purpose bioinformatics environment within the JavaScript infrastructure, similarly to projects such as biopython or bioruby in Python and Ruby, respectively.

The JavaScript infrastructure attracts the attention of developers aside from bioinformaticians, and the D3.js project (Bostock et al. 2011) should be considered as a universal framework for development of online applications, such as 
those used for the semantic analysis and text mining (Borke and Härdle 2016), and in healthcare (Schroeder et al. 2017). The tools provided by D3.js are comparable in efficiency with universal graphics libraries used in Python and R environments (matplotlib, ggplot2), and these libraries are often used to prepare publication-quality images in microbiology research projects.

\section{Materials and methods}

\section{Overview}

The design of the D3b system was composed from several parts: JavaScript-based tools for data presentation on a client side, back-end tools for data processing on the server side, and a web framework on the side of the hub server which manages user queries between the client and server sides. The instruments from the D3.js library were used to generate expressive images based on the input data and the user queries defined as html-based input forms. The Python environment was chosen as an infrastructure for development of tools for data processing on the server side, and this allows us to use functionality of both the scikit-bio library with the function specific to microbial ecology, and general-purpose libraries, such as scipy and sklearn.

Since the resources which could be directed to development of the D3b package were limited, the project was not aimed to compete with packages where a lot of effort had been directed to support the consistency of the project with other packages within the infrastructure. The project is deposited in github in source codes as an installable package (sferanchuk/d3b_charts), however, the installation of the package might require additional efforts due to inconsistencies with updates and modifications introduced in the new releases of the dependencies used in the package.

Instead, the aim of this project was to satisfy the requirements from a local community of microbiologists from the Irkutsk scientific center and related organizations; therefore, the on-line version of the project is continuously supported at the bri-shur.com site. The bri-shur project has a long enough history, and, by design, the web-interface in that project is separated from the servers rendering data processing (Feranchuk et al. 2012). Therefore, in the present version, the infrastructure used in a hub server is implemented within the django framework in Python. However, it is relatively independent from both sides of the system and could be easily substituted by another type of the web framework in further development or adaptation of the project.

Rendering of graphics in the JavaScript framework is implemented in the user browser, however, the stable and consistent functionality for export of graphics in raster and vector formats is obligatory for that kind of the system. Therefore, the phantomjs command-line tool was adopted to run on the server side, as a converter of dynamic html pages to PNG and SVG formats. Export of the tables generated on-line is possible using conventional functionality of the browser and, in addition, export to the tab-delimited format is supported in some cases.

Input data for the analysis, in the form of abundance tables, can be supplied in a biom format and tab-delimited format. When the input file is submitted, access becomes available in the menu of several tools which could be used to analyze the submitted data from different viewpoints. The privacy of the data is supported by assigning a unique url with a secure 32-byte key to access a page with any of data tables. To be able to specify any subset of rows and columns of the submitted table, two types of descriptors could be assigned on-line, and stored together with an input table. The first kind of the descriptor, in the form of tag-value pairs, allows the selection of traits associated with each of the samples in the survey. The second type of the descriptor, in the form of a list of taxonomic identifiers, allows the analysis to be focused on specific taxonomic units, rather than on the whole content of the microbiome.

The figures below illustrating the services described are based on the surveys described in (Feranchuk et al. 2018). Namely, one of the surveys presents bacterial symbionts of marine sponges in coral reefs near an Indonesian shore, as described in (Cleary et al. 2018). The second survey presents the gut microbiome of Anser indicus (Latham, 1790) geese with different breeding patterns, as described in (Wang et al. 2016). The third survey presents the microbiome of jaw bone osteomyelitis in patients with two types of the disease, as described in (Goda et al. 2014).

\section{Specifications}

Estimates of the alpha-diversity adopted in the system are based on implementations from the scikit-bio package (Shannon, Simpson, Chao1, Ace, Fisher $\alpha$, Gini). In addition, an alternative estimator of the Gini measure and two parametric diversity measures, as specified in (Feranchuk et al. 2018), are included in the set of estimators. The rarefaction analysis uses the Python code, adapted from the scikit-bio package, and the ability to estimate the MichaelisMenthen fit to rarefaction curves is included as an alphadiversity estimator.

A set of metrics for distances between samples include weighted and unweighted Unifrac measures, as these are implemented in the scikit-bio package, and a set of metrics, composed of Bray-Curtis similarity, Jaccard similarity, and Euclidean distance, as these are implemented in the scipy package. Measures of Pearson, Spearman, and Kendall correlations are transformed into distances just by subtracting values of the correlation from its maximal value: $d=2-c$. In addition, the Morisita-Horn measure is included in the set of metrics, using a Python code, incorporated in the system.

These distances could be used to construct dendrograms of proximity between samples, to run Permanova and Anosim tests which are implemented in the scikit-bio package, and to run the principal coordinate analysis, the correspondence analysis (implemented in scikit-bio package), the principal 
components analysis or the multi-dimensional scaling (implemented in the sklearn package).

\section{Results}

\section{Overview}

Tabular presentations of the data include a table of abundances, as it is loaded into the system, and with various options for sorting the rows, merging the columns, reducing the level of taxonomic hierarchy, and others. Similar options are available for most of the services within the interactive system, where the interactive features of the $\mathrm{d} 3$ library are used. Namely, most of the input forms include the option of the level of taxonomic hierarchy, the possibility to restrict the analysis or data presentation to certain taxonomic groups, and the possibility to merge samples into pre-defined groups.

The tabular presentations also include:

1) Values of the alpha-diversity calculated using several of the most informative estimators.

2) Significance of differences for alpha-diversity values between several groups, calculated following the methods, described in (Feranchuk et al. 2018).

3) Significance of differences between groups of samples, calculated using the distances between samples, with several alternative measures of distance.

The graphical presentations, implemented with the use of d3.js library, include following charts:

1) A bubble chart and a heatmap, to represent absolute/ relative abundances.

2) $2 \mathrm{D}$ scatter charts, to represent the results of several data ordination methods, such as PCA or multi-dimensional scaling (MDS). The choice of several measures is available here to calculate distances between the samples.

3) A dendrogram (tree) to represent the degree of proximity between samples.

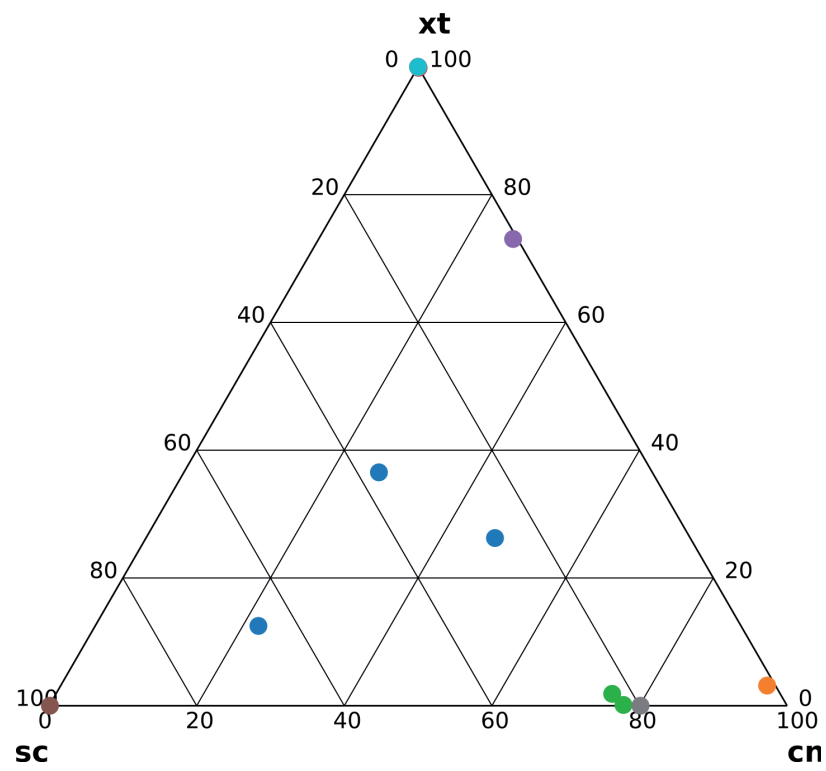

4) A Venn diagram to represent the unique and shared taxonomic units for the samples, implemented with the use of the jVenn (Bardou et al. 2014) plugin and venn.js library.

5) Two kinds of diagrams to present distributions which describe a sample or a group of samples: a rank-abundance chart (Whittaker plot) to represent the distribution of relative species abundance, and a rarefaction curve to estimate the effect of insufficient coverage and the sample size.

6) A ternary chart, to represent the relative abundances of bacterial phylotypes for three samples or groups of samples.

7) A volcano chart and a mean-distance plot, to represent distribution of abundances and differentiation between traits.

8) Two combined 2D charts, to represent the results of PCA decomposition, applied directly to a non-square matrix of abundances. One chart is for samples in the survey and the second adjacent chart is for bacterial species in rows of the submitted matrix.

Case studies

Figure 2 illustrates the variations in microbial communities for different families of marine sponges, at the class level. The colors in Figure 2 are assigned at the level of phyla, the most general category of taxonomy. The presented result confirms both the original study (Cleary et al. 2018) and the wider research where the composition of sponge microbiomes was compared (Thomas et al. 2016). In the second paper, the relative composition of microbiomes was also demonstrated in Figure 3 at the level the phyla, using the heatmap chart. In particular, the Proteobacteria phylum is the most abundant in all sponge families, which is demonstrated in from Figure 2 and in the cited paper.

Figure 2 shows that several classes are specific to Xestospongia Laubenfels, 1932 sponge, and this is confirmed in Table 1, where the values of biodiversity are presented at the phylum level. This table was also generated using online tools, included in the described system. The significance for

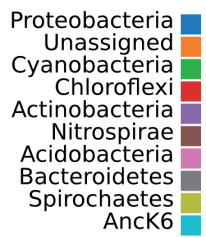

Figure 2. The quantitative composition of symbionts for three types of marine sponges at the level of bacterial phyla, presented as a ternary chart. Species of sponges are abbreviated as follows: Xt, Xestospongia testudinaria (Haplosclerida order); sc, Stylissa carteri (Halichondrida order); cn, Cinachyrella (Spirophorida order). 
Table 1. For the t-test and Mann-Whitney rank-sum test, the minimum p-value, from six combinations of traits is shown. Species of sponges are abbreviated as follows: ap, Aaptos suberitoides (Suberitida order); xt, Xestospongia testudinaria (Haplosclerida order); sc, Stylissa carteri (Halichondrida order); c, Cinachyrella (Spirophorida order).

\begin{tabular}{lcccccccc}
\hline \multicolumn{1}{c}{$*$} & Species & Shannon & Simpson & Fisher Alpha & OTU Number & Chao1 & Ace & Gini \\
\hline PapBSAp1Mer1 & $\mathrm{Ap}$ & 1.8 & 0.64 & 1.82 & 14 & 14.25 & 15.9 & 0.87 \\
PapBSAp1Mer2 & $\mathrm{Ap}$ & 1.89 & 0.66 & 1.58 & 10 & 10.5 & 12.61 & 0.78 \\
PapBSAp1Mer5 & $\mathrm{Ap}$ & 1.68 & 0.6 & 1.18 & 8 & 8 & 9.11 & 0.79 \\
PapBSCn1Kr02 & $\mathrm{Cn}$ & 1.18 & 0.44 & 0.95 & 7 & 7 & 7 & 0.87 \\
PapBSCn1Ms17 & $\mathrm{Cn}$ & 1.41 & 0.55 & 0.9 & 7 & 7 & 7.5 & 0.81 \\
PapBSCn2Kr02 & $\mathrm{Cn}$ & 1.32 & 0.56 & 0.51 & 4 & 4 & 4 & 0.62 \\
PapBSCn2Ms17 & $\mathrm{Cn}$ & 1.93 & 0.71 & 1.19 & 10 & 10 & 10 & 0.76 \\
PapBSCn3Kr02 & $\mathrm{Cn}$ & 2.06 & 0.7 & 1.73 & 13 & 13 & 13.76 & 0.82 \\
PapBSCn3Ms17 & $\mathrm{Cn}$ & 1.99 & 0.72 & 1.1 & 9 & 9 & 9 & 0.71 \\
PapBSSc1Mer1 & $\mathrm{Sc}$ & 0.93 & 0.32 & 0.85 & 6 & 6 & 6 & 0.89 \\
PapBSSc1Mer2 & $\mathrm{Sc}$ & 0.94 & 0.32 & 0.92 & 7 & 8 & 10.9 & 0.9 \\
PapBSSc1Mer5 & $\mathrm{Sc}$ & 1.18 & 0.51 & 0.67 & 5 & 5 & 5 & 0.78 \\
PapBSXt1Mer2 & $\mathrm{Xt}$ & 2.11 & 0.71 & 1.41 & 11 & 11 & 11 & 0.77 \\
PapBSXt1Mer5 & $\mathrm{Xt}$ & 2.29 & 0.74 & 1.88 & 10 & 10 & 10 & 0.7 \\
PapBSXt2Mer1 & $\mathrm{Xt}$ & 2.26 & 0.74 & 1.54 & 11 & 11 & 11 & 0.73 \\
Student's t-test (min. value) & $\mathrm{p}-\mathrm{value}$ & $<0.001$ & $<0.001$ & 0.002 & 0.017 & 0.013 & 0.009 & 0.041 \\
Mann-Whitney test (min. & & 0.014 & 0.026 & 0.04 & 0.038 & 0.038 & 0.078 & 0.04 \\
value) & & 0.002 & 0.005 & 0.03 & 0.119 & 0.147 & 0.179 & 0.172 \\
ANOVA test & & & & & & & 5
\end{tabular}

Figure 3. The composition of the gut microbiome of Anser indicus at the level of bacterial classes, presented as a bubble chart. Types of breeding are abbreviated as follows: a, artificial breeding; w, wild type breeding; sa, semi-artificial breeding

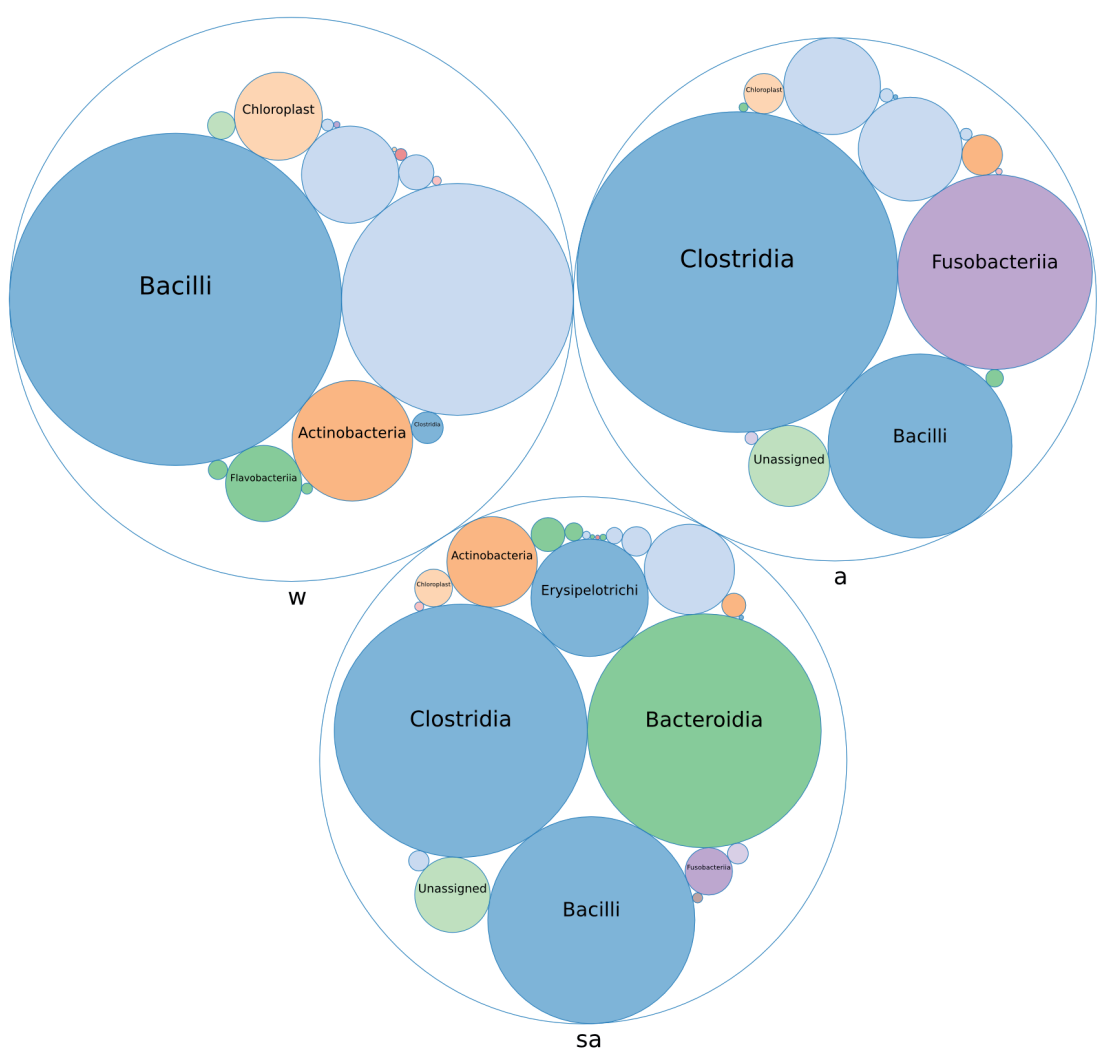

Firmicutes Proteobacteria Bacteroidetes Cyanobacteria 


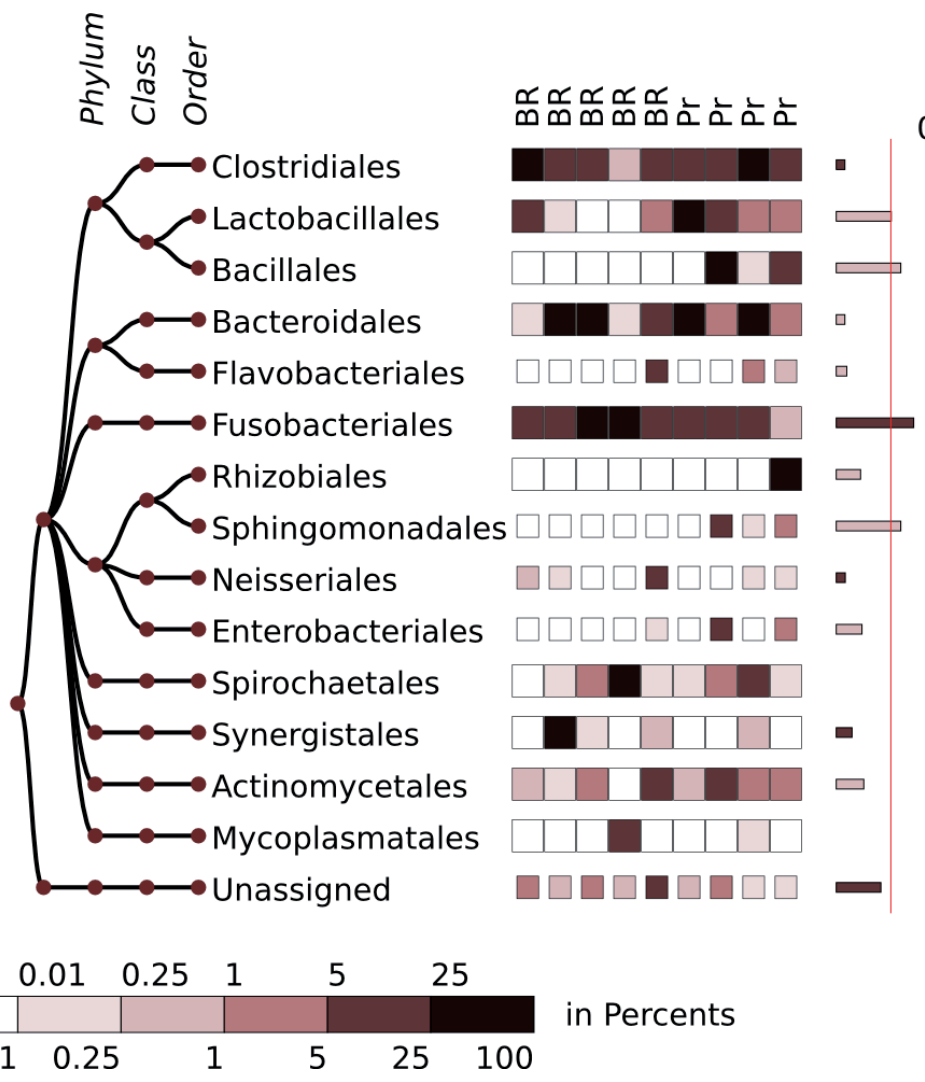

Figure 4. The composition of the microbiome in jaw bone osteomyelitis, presented as a heatmap chart. The 10 most abundant bacterial orders are shown. The orders present with an average abundance $>2 \%$ are highlighted. The scale of the heatmap indicates the abundance values as a percentage. The bars on the right show the degree of separation between two groups for each of the presented phylotypes. The width of the bars is proportional to the $-\log$ (p-value) for the Mann-Whitney test. Types of sub-diagnosis are abbreviated as follows: Pr, primary osteomyelitis; BR, bisphosphonate-related osteonecrosis of the jaw.

\begin{tabular}{l|llllllll}
0 & 0.01 & 0.25 & 1 & 5 & 25 & \\
\hline & & & & & & & \\
in Percents
\end{tabular}

separation of the samples is also shown in Table 1, using three statistical tests, applied to lists of diversity values.

Figure 3 illustrates significant differences in the composition of gut microbiome of $A$. indicus geese, depending on the breeding pattern. In the original study (Wang et al. 2016), a result, similar to the one shown in Figure 3, was presented in Figure 4B using bar charts.

Figure 4 demonstrates composition of the microbiome in inflamed jaw bones, in an agreement with Figure 1 in the original study (Goda et al. 2014), where a similar result was presented using a bar chart. In the cited study the emphasis was placed on a wide diversity of microbiomes in the inflamed bones, with a prevalence of anaerobic bacteria for most samples from patients with different diagnoses. The presentation in Figure 4 might also confirm this conclusion. Although the relative presence of abundant phylotypes might vary in the different samples, no specific bacterial phylotype was specific to each of the sub-diagnoses with a sufficient confidence.

\section{Discussion}

With richness of the material and a wide spectrum of possible applied results, the scientific research in microbiology becomes, to a great extent, a creative work rather than routine investigation or description of the observed phenomenon within pre-defined rigorous templates. In addition, as it is common for any genre of creative work, a niche could easily be found to motivate development of any concept or idea, even if it has an unlikely chance to generate a direct profit of any kind. The same is true for development of a software for microbiologists.

Recently, microbial ecology got to a new level of its development, due to appearance of easy and highly effective methods to study bacterial communities. Since the relation between price and quality has drastically changed in favor of researchers, the amplicon sequencing is available to almost everyone. But a qualitative analysis of data, obtained on a stage of sequencing, strongly depends on bioinformatics resources, which are used for an analysis. For a specialist in microbial ecology, some resources with unified algorithms are required, so that one may do a kind of creative work: to vary groups of samples in the underlying dataset, to introduce new criteria of inclusions or exclusions to groups of samples, to look up for trends and specifics in composition, structure and functions of microbial communities. At the moment, a number of software and services are available which allows to perform correct processing of amplicon libraries (RDP, Greengenes, QIIME), to run a deep comparative analysis (QIIME) or predictive analysis of functions (Picrust), but most of these services and packages require sufficient skills in bioinformatics.

An advantage of the D3b system is to provide possibility for creative work even for a user with no specific training in bioinformatics. At first, it could be of interest to healthcare workers, who could rely on the data from amplicon sequencing, e.g., samples of the gut microbiome, in a personalized approach to plan diagnostics, prevention or treatment of diseases. 
The system might be not sufficient or satisfactory to fit some templates which would allow it to be classified as a mature and consistent software project. It is in part a consequence of connection of several auxiliary libraries in one package, so that installation of the package could be unstable. Also, as rendering of the data is implemented on the server side, the interactive functionality of D3 library is limited to pop-up tooltips and to the page with assignment of taxonomic filters.

The primary aim in development of the system was to provide a tool to prepare publication-ready images. And the system has a simple and easy-to-learn interface for grouping of data and for introduction of new criteria for analysis. In particular, the ability to assign taxonomy filtering allows to analyze target groups of bacteria in all the samples; it could arose from different taxons but compose a reference microflora in the gut microbiome.

The tables of variations between indices of the alpha-diversity and distance measure provide an easy way to present the results, relying on a significance of separation between groups of samples. At the same time, the list of charts present almost a complete set of graphical presentations, developed at the moment, and allow easy ways to present both individual samples, and samples merged by certain traits.

And the presented system itself can be also described as a completed kind of creative work in a genre of software development. An expressiveness and usability - criteria used to estimate the value of a software tool - could be supported in the microbiology software in many ways, and a way provided in the D3b system, as we suppose, has a right to be considered at the same level as the most widely used brand names of similar applications.

Conflict of interest. The authors declare that there is no conflict of interest.

Acknowledgements: The authors appreciate the contributions from the specialists from NIPCHI, LIN, SIFIBR, and SFU to the development of the system, and are personally grateful to L. Mironova, A. Ponomaryova, A. Gladkikh, A. Krasnopeev, Y. Putintseva, Y. Markova, and I. Petrushin. S.F. and U.P. thank C.H. Brown for a long-term support of their research activity.

\section{References}

Almeida, A., A.L. Mitchell, A. Tarkowska and R.D. Finn. 2018. Benchmarking taxonomic assignments based on 16S rRNA gene profiling of the microbiota from commonly sampled environments. GigaScience 7(5):1-10.

Bardou, P., J. Mariette, F. Escudie, C. Djemiel and C. Klopp. 2014. jvenn: an interactive Venn diagram viewer. BMC Bioinformatics 15(1):293.

Borke, L. and W. Härdle. 2016. Q3-D3-LSA, SFB 649 Discussion Paper, No. 2016-049, SFB 649, Economic Risk, Berlin.

Bostock, M., V. Ogievetsky and J. Heer. 2011. D3: data-driven documents. IEEE Trans. Vis. Comput. Graph. 17(12):2301-2309.
Boughner, L.A. and P. Singh. 2016. Microbial ecology: where are we now? Postdoc. J. 4(11):3-17.

Caporaso, J.G., J. Kuczynski, J. Stombaugh, K. Bittinger, F.D. Bushman, E.K. Costello, N. Fierer, A.G. Pena, J.K. Goodrich, J.I. Gordon, G.A. Huttley, S.T. Kelley, D. Knights, J.E. Koenig, R.E. Ley, C.A. Lozupone, D. McDonald, B.D. Muegge, M. Pirrung, J. Reeder, J.R. Sevinsky, P.J. Turnbaugh, W.A. Walters, J. Widmann, T. Yatsunenko, J. Zaneveld and R. Knight. 2010. QIIME allows analysis of high-throughput community sequencing data. Nat. Methods 7:335-336.

Cleary, D.F.R., A.R.M. Polonia, L.E. Becking, N.J. de Voogd, P.H. Gomes and N.C.M. Gomes. 2018. Compositional analysis of bacterial communities in seawater, sediment, and sponges in the Misool coral reef system, Indonesia. Mar. Biodiv. 48:1889.

Cole, J.R., Q. Wang, J.A. Fish, B. Chai, D.M. McGarrell, Y. Sun, C.T. Brown, A. Porras-Alfaro, C.R. Kuske and J.M. Tiedje. 2014. Ribosomal database project: data and tools for high throughput rRNA analysis. Nucl. Acids Res. 42:D633-D642.

Feranchuk, S., N. Belkova, U. Potapova, D. Kuzmin and S. Belikov. 2018. Evaluating the use of diversity indices to distinguish between microbial communities with different traits. Res. Microbiol. 169:254-261.

Feranchuk, S., U. Potapova, V. Potapov, D. Mukha, V. Nikolaev and S. Belikov. 2012. Tools for protein structure prediction at the brishur.com web portal. J. Life Sci. 6:1074-1079.

Goda, A., F. Maruyama, Y. Michi, I. Nakagawa and K. Harada. 2014. Analysis of the factors affecting the formation of the microbiome associated with chronic osteomyelitis of the jaw. Clin. Microbiol. Infect. 20(5):O309-317.

Hugerth, L.W. and A.F. Andersson. 2017. Analysing microbial community composition through amplicon sequencing: from sampling to hypothesis testing. Front. Microbiol. 8:1561.

Kopylova, E., J.A. Navas-Molina, C. Mercier, Z.Z. Xu, F. Mahe, Y. He, H.W. Zhou, T. Rognes, J.G. Caporaso and R. Knight. 2016. Open-source sequence clustering methods improve the state of the art. mSystems 1(1):pii:e00003-15.

McDonald, D., J.C. Clemente, J. Kuczynski, J.R. Rideout, J. Stombaugh, D. Wendel, A. Wilke, S. Huse, J. Hufnagle, F. Meyer, R. Knight and J.G. Caporaso. 2012. The Biological Observation Matrix (BIOM) format or: how I learned to stop worrying and love the ome-ome. GigaScience 1(1):7.

Oksanen, J., R. Kindt, P. Legendre, B. O'Hara, M.H.H. Stevens and M.J. Oksanen. 2007. The vegan package. Community ecology package. 10:631-637.

Pettersen, E.F., T.D. Goddard, C.C. Huang, G.S. Couch, D.M. Greenblatt, E.C Meng and T.E. Ferrin. 2004. UCSF Chimera-a visualization system for exploratory research and analysis. $J$. Comput. Chem. 25(13):1605-1612.

Schloss, P.D., S.L. Westcott, T. Ryabin, J.R. Hall, M. Hatmann, E.B. Hollister, R.A. Lesniewski, B.B. Oakley, D.H. Parks, C.J. Robinson, J.W. Sahl, B. Stres, G.G. Thallinger, D.J. Van Horn and C.F. Weber. 2009. Introducing Mothur: open-source, platform-independent community-supported software for describing and comparing microbial communities. Appl. Environ. Microbiol. 75:7537-7541.

Schroeder, J., J. Hoffswell, C.F. Chung, J. Fogarty, S. Munson and J. Zia. 2017. Supporting patient-provider collaboration to identify individual triggers using food and symptom journals. CSCW Conf. Comput. Support Coop. Work 2017:1726-1739.

Segata, N., D. Boernigen, T.L. Tickle, X.C. Morgan, W.S. Garrett and C. Huttenhower. 2013. Computational meta'omics for microbial community studies. Mol. Syst. Biol. 9:666. 
The PyMOL molecular graphics system. 2017. Version 2.0, Schrodinger LLC

Thomas, T., L. Moitinho-Silva, M. Lurgi, J.R. Bjork, C. Easson, C. Astudillo-Garcia, J.B. Olson, P.M. Erwin, S. Lopez-Legentil, H. Luter, A. Chaves-Fonnegra, R. Costa, P.J. Schupp, L. Steindler, D. Erpenbeck, J. Gilbert, R. Knight, G. Ackermann, J. Victor Lopez, M.W. Taylor, R.W. Thacker, J.M. Montoya, U. Hentschel and N.S. Webster. 2016. Diversity, structure and convergent evolution of the global sponge microbiome. Nat. Commun. 7:11870.

Wang, W., J. Cao, J.R. Li, F. Yang, Z. Li and L.X. Li. 2016. Comparative analysis of the gastrointestinal microbial communities of bar-headed goose (Anser indicus) in different breed- ing patterns by high-throughput sequencing. Microbiol. Res. 182:59-67.

Yachdav, G., T. Goldberg, S. Wilzbach, D. Dao, I. Shih, S. Choudhary, S. Crouch, M. Franz, A. Garcia, L.J. Garcia, B.A. Gruning, D. Inupakutika, I. Sillitoe, A.S. Thanki, B. Vieira, J.M. Villaveces, M.V. Schneider, S. Lewis, S. Pettifer, B. Rost and M. Corpas. 2015. Anatomy of BioJS, an open source community for the life sciences. Elife 8:4.

Received March 22, 2019

Revised August 23, September 16, 2019 Accepted September 22, 2019 